\title{
Dietary iron intake and Type 2 diabetes incidence in postmenopausal women: the lowa Women's Health Study
}

\author{
D.-H. Lee ${ }^{1,2}$ • A. R. Folsom ${ }^{1}$ D. R. Jacobs ${ }^{1,3}$ \\ ${ }^{1}$ Division of Epidemiology, School of Public Health, University of Minnesota, Minneapolis, USA \\ ${ }^{2}$ Department of Preventive Medicine, College of Medicine, Kosin University, Pusan, Korea \\ ${ }^{3}$ Institute for Nutrition Research, University of Oslo, Oslo, Norway
}

\section{Abstract}

Aims/hypothesis. Recently, a clear biological link between iron metabolism and diabetes has emerged from epidemiological and experimental studies. We carried out a prospective study of dietary iron intake and incidence of Type 2 diabetes.

Methods. 35,698 postmenopausal women initially aged 55 to 69 years were followed for 11 years. Diet was assessed with a food frequency questionnaire at baseline.

Results. Intake of heme iron showed a positive association with incident Type 2 diabetes; the relative risks were $1.0,1.07,1.12,1.14$, and 1.28 across quintiles of heme iron ( $p$ trend $=0.02$ ) after adjustment for non-dietary and dietary risk factors. Heme iron showed a weak positive association among non-drinkers, but the association appeared to be stronger among subjects who consumed more alcohol. For example, in a model restricted to those who drank alcohol at least $15 \mathrm{~g} /$ day, adjusted relative risks across quintiles of heme iron were $1.0,2.26,3.22,1.92$, and 4.42 ( $p$ trend $=0.05$ ); and consumers of $30 \mathrm{~g} /$ day of more of supplemental iron had an adjusted relative risk equal to 3.03 (95\% CI, 1.29-7.12)], compared to those who took no iron supplement. Non-heme iron was inversely associated with incidence of Type 2 diabetes. Amongst nondrinkers adjusted relative risks were 1.0, 0.83, 0.87, 0.72 , and 0.67 across quintiles $(p$ trend $<0.01$ ). This inverse association was lost among drinkers, in whom there was no association of diabetes incidence with non-heme iron.

Conclusions/interpretation. Greater dietary heme-iron intake and/or supplemental iron were associated with an increased risk of Type 2 diabetes, especially amongst those who drink alcohol. [Diabetologia (2004) 47:185-194]

Keywords Type 2 diabetes $\cdot$ Iron · Alcohol consumption · Meat intake
Epidemiological studies have reported a positive relationship of serum ferritin with several components of the insulin resistance syndrome and with an elevated

Received: 7 July 2003 / Revised: 21 October 2003

Published online: 8 January 2004

(C) Springer-Verlag 2004

D. R. Jacobs Jr (๘)

Division of Epidemiology, School of Public Health,

University of Minnesota, 1300 South 2nd Street, Suite 300,

Minneapolis, MN 55454 USA

E-mail: jacobs@epi.umn.edu

Abbreviations: WHR, waist-to-hip ratio $\cdot \mathrm{RR}$, relative risk . Q1-Q5, quintiles 1-5 risk of Type 2 diabetes [1, 2, 3, 4, 5]. In addition, low iron status or iron depletion is associated with better insulin action and metabolic control in healthy individuals and Type 2 diabetic patients [6, 7, 8]. Hemochromatosis or the hemochromatosis genotype also has been associated with the risk of Type 2 diabetes [9], though not consistently [10]. A recent review paper [11] has made a clear biological link between iron metabolism and diabetes based on both epidemiologic and experimental studies. Therefore, there is a possibility that dietary iron could influence risk of diabetes.

The human body has several defence mechanisms to protect against any harmful effect of free iron; for example, careful regulation of iron absorption and iron-binding proteins $[12,13]$. However, alcohol con- 
sumption has been reported to disrupt iron homeostasis through effects on iron binding proteins such as ferritin and transferrin [14], even in the general population $[15,16,17]$. Therefore, if there is an effect of iron intake on the development of diabetes, the effect of iron might occur mostly amongst drinkers.

Therefore, we carried out an analysis in the Iowa Women's Health Study to examine (i) if there is a relation between dietary iron intake and incidence of Type 2 diabetes over 11 years of follow-up and (ii) if the association between dietary iron intake and Type 2 diabetes is different depending on alcohol consumption.

\section{Subjects and methods}

The Iowa Women's Health Study cohort. The study was approved by the Committee on the Use of Human Subjects in Research, University of Minnesota; women signed a consent form prior to beginning participation in the study. Methods for the Iowa Women's Health Study recruitment and data collection have been published previously [18]. Briefly, this study was designed to examine associations between several host, dietary, and lifestyle factors and the incidence of cancer in 41,836 postmenopausal women, aged 55 to 69 years at baseline, who completed a 16-page self-administered questionnaire in January 1986. Women were excluded from analysis if they reported implausibly high $(>5000 \mathrm{kcal})$ or low $(<600 \mathrm{kcal})$ energy intakes, left more than or equal to 30 items blank on the foodfrequency questionnaire, were premenopausal, or reported diabetes at baseline. Women were considered to have diabetes at baseline if they responded "yes" to one of the following questions: (i) Have you ever been told by a doctor that you have sugar diabetes? (ii) Have you ever taken insulin or pills for sugar diabetes (or to lower blood glucose)? After exclusions, 35,698 women remained eligible for the study.

Data collection. The baseline questionnaire included questions on known or suspected risk factors for diabetes, such as age, BMI, waist-to-hip ratio (WHR), physical activity, alcohol consumption, and smoking history. BMI was calculated from weight and height measurements provided by the participants. WHR was calculated as the average of two measurements taken by the participant's spouse or friend using a paper tape measure that was included with the questionnaire. The women reported their frequency of moderate (e.g., golf and long walks) and vigorous (e.g., swimming and aerobics) physical activity and a three level score was created combining frequency and intensity. Pack-years of smoking were calculated from information on the intensity and duration of cigarette smoking. Alcohol consumption was assessed with a food frequency questionnaire that queried the participants' typical intakes of wine, beer, and spirits. In addition, the participants provided information on their marital status, educational attainment, residence, and use of hormone replacement therapy.

A 127-item food-frequency questionnaire similar to that used in the 1984 survey of the Nurses' Health Study was used to assess typical food intake over the previous year [19, 20]. Nutrient intake was computed by multiplying the frequency response by the nutrient content of the specified portion sizes. Content of heme iron was calculated by applying a factor of 0.4 to the total iron content of all meat items [21]. Non-heme iron was computed as total iron in non-meat foods and 0.6 of the total iron content in meats [21]. We also asked questions about iron and multivitamin supplements. The iron content of multivitamin or iron supplements was calculated based on brand.

Type 2 diabetes incidence was determined by an affirmative response to the following question, which was on all of the follow-up mailed surveys: "Since baseline (or last follow-up), were you diagnosed for the first time by a doctor as having sugar diabetes?". During 11 years of follow-up, 1921 women reported incidence of Type 2 diabetes in the four follow-up surveys administered in $1987(n=365), 1989 \quad(n=338), 1992$ $(n=471)$, and $1997(n=747)$. Response rates for the four follow-up surveys were $91,89,86$, and $79 \%$, respectively.

Data analysis. For women who did not report a diagnosis of incident Type 2 diabetes, person-time-at-risk was calculated from baseline to the date of the last completed follow-up survey. For women who reported an incident diagnosis of Type 2 diabetes, person-time was calculated as the sum of the known disease-free period plus half of the period during which the diagnosis was made. Mortality status was determined annually through linkage with the State Health Registry of Iowa or, in the case of non-respondents and emigrants from Iowa, via the National Death Index.

Total dietary iron, dietary non-heme iron, dietary heme iron, and supplemental iron were examined as exposures. Participants were categorized according to quintiles of dietary iron intake. Supplemental iron was classified as $0 \mathrm{~g} / \mathrm{d}, 1$ to $29 \mathrm{~g} / \mathrm{d}$, and more than or equal to $30 \mathrm{~g} / \mathrm{d}$. Results were displayed among all subjects and also stratified by alcohol consumption $(0 \mathrm{~g} / \mathrm{d}, 1-14 \mathrm{~g} / \mathrm{d}$, and $\geq 15 \mathrm{~g} /$ day $)$. In the Iowa Women's Health Study, information on alcohol intake was collected twice, in 1986 and 1992. In the present analyses, for women who had an event or were last followed up before 1992, we used alcohol consumption based on the 1986 questionnaire and for women who had an event or were last followed up after 1994, we used averaged alcohol intake based on both 1986 and 1994.

We used proportional hazards regression to account for potential confounding effects of other risk factors for Type 2 diabetes. The independent variable of interest was either as total dietary, dietary non-heme, dietary heme, or supplemental iron. Multivariate models were adjusted simultaneously for age (continuous), total energy intake (continuous), WHR (continuous), BMI (continuous), physical activity score (low, medium, or high), cigarette smoking (none, 1-19 pack-years, 20-39 pack-years, or $\geq 40$ pack-years), education (no high school diploma, high school diploma, college or vocational school but no degree, or college degree), marital status (currently married, never married, separated or divorced, or widowed), residential area (farm, rural or small town with population up to 2499 , town of population from $2500-10,000$, or city or town with population $>10,000$ ), and hormone replacement therapy (current, former, or never), plus a continuous variable for alcohol (g/day). An additional model adjusted for animal fat (continuous), vegetable fat (continuous), cereal fibre (continuous), and dietary magnesium (continuous) and also included dietary nonheme, dietary heme, and supplemental iron simultaneously. In tests for trend, the quintile number of dietary iron intake was used as a continuous variable. For all relative risks (RR), we calculated $95 \%$ CI. All $p$ values were two-tailed and less than or equal to 0.05 was considered to be statistically significant.

\section{Results}

Most of total dietary iron (91\%) was from non-heme iron and there was a weak inverse association between 
Table 1. Age and total energy intake-adjusted ${ }^{\mathrm{a}}$ mean levels of diabetes risk factors according to extreme quintiles (Q1 lowest and Q5 highest) of dietary iron intake and by supplemental iron at baseline in the Iowa Women's Health Study, 1986

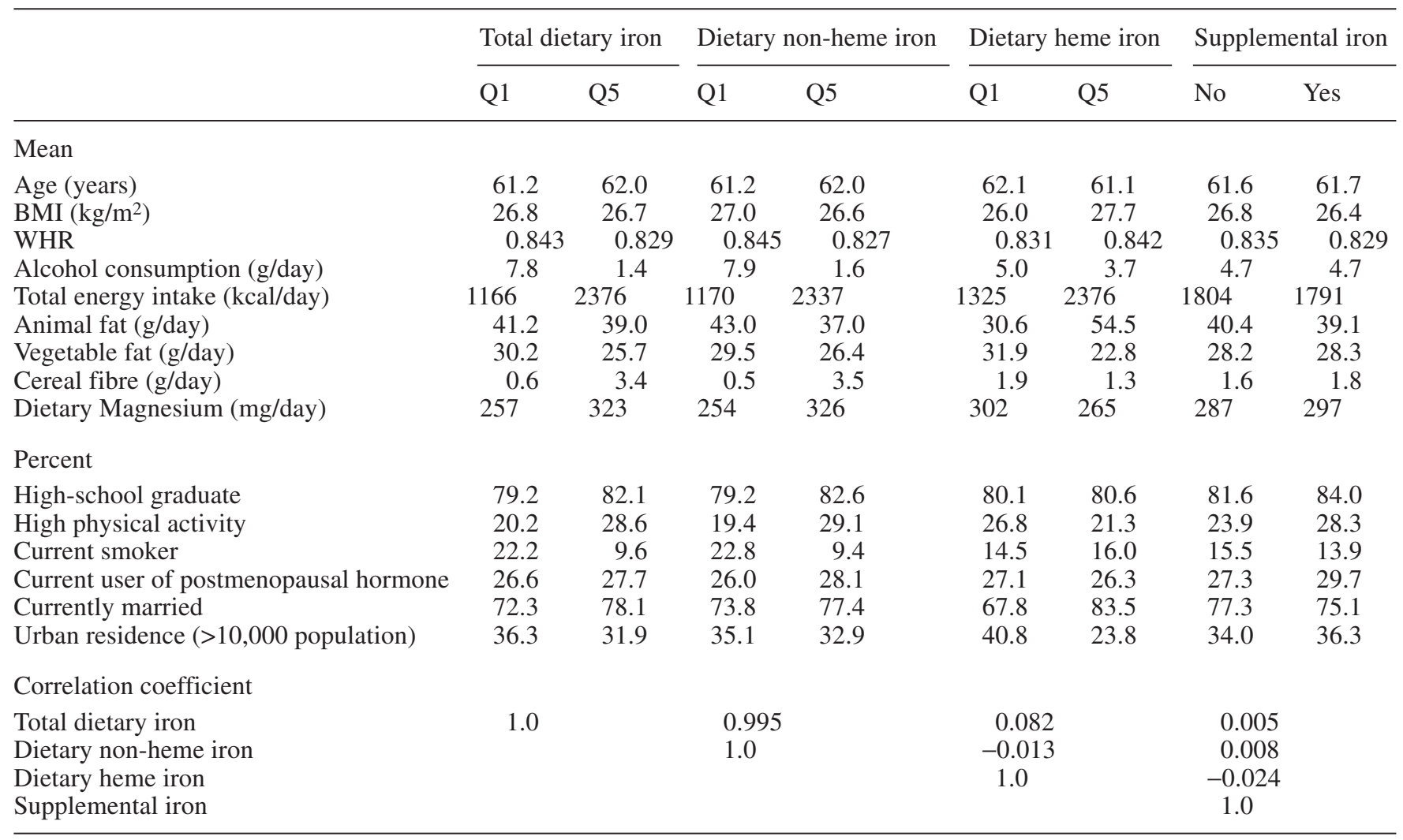

${ }^{a}$ Mean levels for age are unadjusted and those for energy intake are age-adjusted

non-heme iron and heme iron consumption (Table 1). Compared to women in the lowest quintile of non-heme iron intake (Q1), women in the highest quintile of nonheme iron intake (Q5) consumed more energy, less animal fat, less vegetable fat, more cereal fibre, and more dietary magnesium. They tended to have healthier behaviour; they had lower BMI and WHR, consumed less alcohol, were less likely to smoke cigarettes, and engaged in more physical activity. Women who consumed more dietary heme iron (Q5 compared to Q1 of heme iron) showed opposite trends. They seemed to have a less healthy diet pattern; they consumed more animal fat, less cereal fibre, and less dietary magnesium. In addition, they had higher mean BMI and WHR, were more likely to smoke cigarettes, and engaged in less physical activity. Supplemental iron was taken by 7006 women, of whom 1408 took more than or equal to $30 \mathrm{~g} / \mathrm{d}$. $88 \%$ of women who consumed supplemental iron at baseline were multivitamin users. Women who took iron supplements had somewhat healthier behaviour and dietary habits than women who did not take supplemental iron. Intakes of iron from non-heme, heme, and supplemental sources were mutually uncorrelated. Total iron intake was largely reflective of non-heme iron intake $(r=0.995)$ and was not analyzed further.

After adjusting for known Type 2 diabetes risk factors and total energy intake, the RRs for quintiles of non-heme dietary iron were $1.0,0.95,0.97,0.86$, and 0.73 ( $p$ trend $<0.01$, Table 2 ). This inverse association could be attributable to other dietary factors, because the association was attenuated and became statistically non-significant after additional adjustment for animal fat, vegetable fat, cereal fibre, and dietary magnesium. Additional adjustment for vitamin $\mathrm{C}$ or calcium, which can affect absorption of non-heme, did not change the results. In contrast, heme iron showed a positive association with adjusted RRs of 1.0, 1.07, $1.12,1.14$, and 1.28 ( $p$ trend $=0.02$ ). Supplemental iron was not associated with incidence of Type 2 diabetes.

Within each level of iron intake a reduction in Type 2 diabetes risk was seen among alcohol drinkers compared to nondrinkers, with further reductions at higher levels of alcohol intake (Table 3A, Table 4A, Table 5A). However, this inverse association with alcohol intake was attenuated as iron intake increased, with the result that a strong inverse gradient with increasing alcohol intake disappeared among those who consumed the most dietary or supplemental iron. After stratification by level of alcohol consumption (Table 3B, Table 4B, Table 5B), dietary non-heme iron was inversely associated with the risk of Type 2 diabetes only among non-drinkers; adjusted RRs for quintile of non-heme iron were $1.0,0.83,0.87,0.72$, 
Table 2. Relative risks of Type 2 diabetes according to intake of dietary non-heme iron, dietary heme iron, supplemental iron in the Iowa Women's Health Study, 1986 to 1997

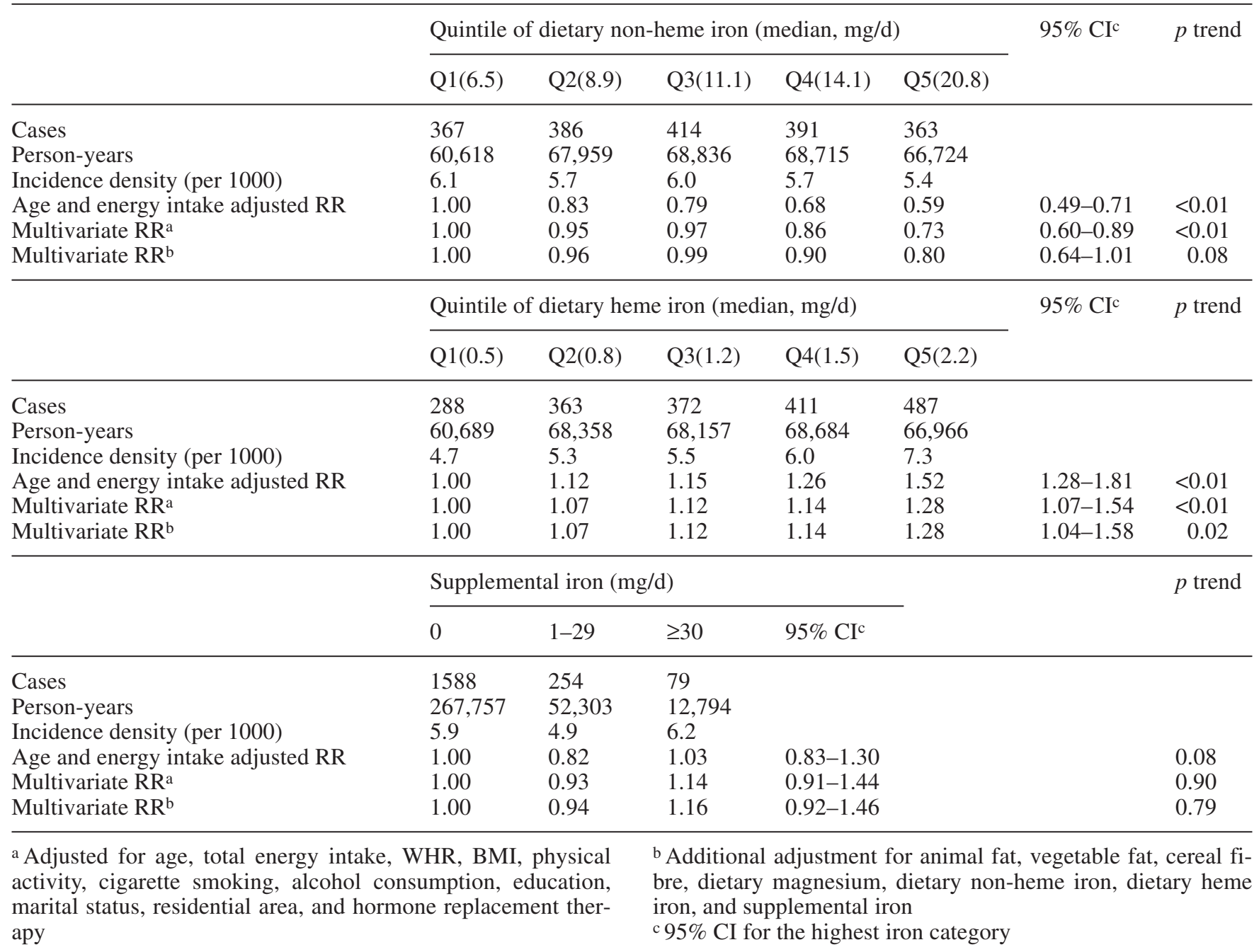

and 0.67 ( $p$ trend <0.01) (Table 3B). Non-heme iron was not associated with Type 2 diabetes among drinkers $(p<0.01$ in Table $3 \mathrm{~A})$. In contrast, heme iron showed a positive association with incident Type 2 diabetes among both non-drinkers and drinkers (Table 4). Furthermore, the heme iron association appeared to be stronger in subjects who reported consuming more drinks per day $(p=0.01$, Table 4A). For example, in a model restricting to drinkers of more than or equal to $15 \mathrm{~g} / \mathrm{d}$, adjusted RRs across quintiles of heme iron were $1.0,2.26,3.22,1.92$, and 4.42 ( $p$ trend $=0.05$, Table 4B). Intake of supplemental iron greater than or equal to $30 \mathrm{mg} / \mathrm{d}$ showed a positive association only among drinkers and the association became stronger among those who drank more (Table 5A) $(p=0.47)$. Women who took supplemental iron more than or equal to $30 \mathrm{~g} / \mathrm{d}$ showed an increased risk of diabetes incidence in a separate regression analysis within drinkers of more than or equal to $15 \mathrm{~g} / \mathrm{d}[\mathrm{aRR}=3.03(95 \% \mathrm{CI}, 1.29-7.12$, Table 5B)].
We also examined the association between red meat intake, the main source of heme-iron, and Type 2 diabetes. Overall, adjusted RRs for quintile of red meat consumption were $1.0,1.01,1.07,1.12$, and 1.20 ( $p$ trend $=0.02$ ). The association became stronger among drinkers of higher amounts of alcohol $(p=0.02$, Table 6A). Neither animal fat nor total dietary saturated fat, both commonly eaten in red meat were associated with incident Type 2 diabetes (data not shown).

\section{Discussion}

Iron is a transition metal that can easily become oxidized and thus act as an oxidant in the body [22]. The general effect of catalytic iron is to convert poorly reactive free radicals, such as $\mathrm{H}_{2} \mathrm{O}_{2}$, into highly reactive ones, such as the hydroxyl radical [22]. A recent review paper has reported influences of iron metabolism on Type 2 diabetes based on experimental and epidemiological studies [11]. The possible mechanisms are 
Table 3. Adjusted ${ }^{\text {a }}$ relative risks of Type 2 diabetes according to intake of dietary non-heme iron stratified by alcohol consumption in the Iowa Women's Health Study, 1986 to 1997

Quintile of non-heme iron(median, mg/d)

\begin{tabular}{lllll}
\hline Q1(6.5) & Q2(8.9) & Q3(11.1) & Q4(14.1) & Q5(20.8)
\end{tabular}

A. Joint model across all drinking categories ( $p$ for interaction $\leq 0.01$ )

Non-drinkers

\begin{tabular}{|c|c|c|c|c|c|}
\hline Cases & 258 & 227 & 245 & 223 & 215 \\
\hline Person-years & 29,962 & 31,438 & 31,760 & 32,321 & 33,001 \\
\hline Incidence density (per 1000) & 8.6 & 7.2 & 7.7 & 6.9 & 6.5 \\
\hline Multivariate RR (95\% CI) & Reference & $0.86(0.71-1.04)$ & $0.90(0.74-1.09)$ & $0.74(0.59-0.92)$ & $0.68(0.53-0.88)$ \\
\hline \multicolumn{6}{|l|}{$1-14 \mathrm{~g} / \mathrm{d}$ alcohol } \\
\hline Cases & 94 & 139 & 155 & 149 & 138 \\
\hline Person-years & 25,359 & 31,049 & 32,310 & 32,291 & 30,668 \\
\hline Incidence density (per 1000) & 3.7 & 4.5 & 4.8 & 4.6 & 4.5 \\
\hline Multivariate RR $(95 \% \mathrm{CI})$ & $0.49(0.38-0.62)$ & $0.60(0.48-0.75)$ & $0.65(0.52-0.81)$ & $0.63(0.49-0.80)$ & $0.57(0.43-0.75)$ \\
\hline \multicolumn{6}{|l|}{$\geq 15 \mathrm{~g} / \mathrm{d}$ alcohol } \\
\hline Cases & 15 & 20 & 14 & 19 & 10 \\
\hline Person-years & 5299 & 5473 & 4767 & 4104 & 3056 \\
\hline Incidence density (per 1000) & 2.8 & 3.7 & 2.9 & 4.6 & 3.3 \\
\hline Multivariate RR (95\% CI) & $0.38(0.22-0.64)$ & $0.48(0.30-0.77)$ & $0.33(0.19-0.59)$ & $0.65(0.40-1.07)$ & $0.37(0.19-0.72)$ \\
\hline
\end{tabular}

B. Separate models within drinking categories

Non-drinkers

Multivariate RR $(95 \%$ CI) 1.0

.0

$0.83(0.69-1.01) \quad 0.87(0.71-1.08) \quad 0.72(0.57-0.92) \quad 0.67(0.50-0.89)$

$p$ trend

$1-14 \mathrm{~g} / \mathrm{d}$ alcohol

Multivariate RR $(95 \% \mathrm{CI}) \quad 1.0$

$1.25(0.94-1.65) \quad 1.32(0.98-1.77) \quad 1.30(0.93-1.82) 1.19(0.80-1.78)$

$\leq 0.01$

$\geq 15$ g/d alcohol

Multivariate RR $(95 \% \mathrm{CI}) \quad 1.0$

$1.13(0.54-2.37) \quad 0.64(0.27-1.53) \quad 1.08(0.42-2.79) \quad 0.49(0.14-1.73)$ $=0.37$

a Adjusted for age, total energy intake, WHR, BMI, physical activity, cigarette smoking, alcohol consumption, education, marital status, residential area, hormone replacement therapy, animal fat, vegetable fat, cereal fibre, dietary magnesium, dietary heme iron and supplemental iron

another US study [23], showed a different relation. Non-heme iron was inversely associated with Type 2 diabetes among non-drinkers, but this apparent advantage disappeared among drinkers.

Even though most dietary iron comes from nonheme sources, heme iron absorption is about five to ten times greater than non-heme iron absorption. The absorption of heme iron is less sensitive to body iron stores and not influenced by other dietary factors [24]. Some population studies showed a positive association between meat intake or heme iron and serum ferritin, a marker of stored body iron, but the association of non-heme iron intake with serum ferritin was negligible $[16,25]$, although the findings have not been totally consistent $[26,27,28]$.

In this cohort, $91 \%$ of total dietary iron was from non-heme sources. Therefore, even considering the low absorption rate of non-heme iron, the absolute amount of available non-heme iron might be similar to consumed higher amounts of alcohol. Non-heme iron, which comprises $91 \%$ of total dietary iron, similar to 
Table 4. Adjusted $^{\mathrm{a}}$ relative risks of Type 2 diabetes according to intake of dietary heme iron stratified by alcohol consumption in the Iowa Women's Health Study, 1986 to 1997

Quintile of heme iron(median, mg/d)

\begin{tabular}{llll}
\hline Q1(0.5) & Q2(0.8) & Q3(1.2) & Q4(1.5)
\end{tabular}

A. Joint model across all drinking categories $(p$ for interaction $=0.01)$

Non-drinkers

$198 \quad 234$

Person-years 32,121

Incidence density (per 1000) 6.2

32,197

7.3

216

Multivariate RR $(95 \% \mathrm{CI}) \quad$ Reference

$1.10(0.91-1.35) \quad 1.11(0.90-1.36) \quad 1.11(0.90-1.37) 1.23(0.97-1.55)$

$1-14 \mathrm{~g} / \mathrm{d}$ alcohol

Cases 85

Person-years 24,798

$85 \quad 116$

Incidence density (per 1000) 3.4

31,643

3.7

32,304

32,304

4.2

216
30,920

7.0

232

288

Multivariate RR $(95 \%$ CI)

$0.68(0.52-0.89) \quad 0.68(0.53-0.86)$

$0.77(0.61-0.98)$

$165 \quad 174$

$32,146 \quad 30,785$

$5.1 \quad 5.7$

$\geq 15$ g/d alcohol

$\begin{array}{llllll}\text { Cases } & 5 & 13 & 21 & 14 & 25 \\ \text { Person-years } & 3772 & 4518 & 4932 & 5152 & 4325 \\ \text { Incidence density (per 1000) } & 1.3 & 2.9 & 4.3 & 2.7 & 5.8 \\ \text { Multivariate RR (95\% CI) } & 0.25(0.10-0.62) & 0.54(0.30-0.95) & 0.74(0.46-1.18) & 0.43(0.25-0.76) & 0.87(0.55-1.36)\end{array}$

B. Separate models within drinking categories

Non-drinkers

Multivariate RR $(95 \%$ CI) 1.

$1.10(0.90-1.35) \quad 1.10(0.89-1.36) \quad 1.11(0.90-1.39) 1.22(0.94-1.58) \quad p$ trend $=0.21$

$1-14 \mathrm{~g} / \mathrm{d}$ alcohol

Multivariate RR $(95 \%$ CI $) \quad 1.0$

$0.95(0.71-1.28) \quad 1.08(0.80-1.45) \quad 1.20(0.89-1.63) 1.20(0.84-1.73) \quad p$ trend

$\geq 15 \mathrm{~g} / \mathrm{d}$ alcohol

Multivariate RR $(95 \%$ CI $) \quad 1.0$

$2.26(0.79-6.44) 3.22(1.16-8.94)$

$1.92(0.63-5.87) 4.42(1.37-14.25)$ $=0.12$

a Adjusted for age, total energy intake, WHR, BMI, physical activity, cigarette smoking, alcohol consumption, education, marital status, residential area, hormone replacement therapy, animal fat, vegetable fat, cereal fibre, dietary magnesium, dietary non-heme iron and supplemental iron that of available heme iron. Currently, there is no evidence that the two types of iron play a different role after absorption in the human body. Other nutrients and unknown components of the main food sources of non-heme iron, such as grains, fruits, and vegetables might explain any beneficial effect of non-heme iron among non-drinkers. Such a prudent diet, characterized by higher consumption of vegetables, fruit, fish, poultry, and whole grains, is associated with a lower risk of Type 2 diabetes [29]. The inverse association between non-heme iron and Type 2 diabetes remained even after adjusting for vegetable fat and cereal fibre, which are known beneficial nutrients contained in the main food sources of non-heme iron [30, 31]. However, these specific dietary factors probably explain only part of the effect of diet on glucose metabolism; a direct effect of dietary non-heme iron is consistent with a disappearance of the inverse association of nonheme iron with Type 2 diabetes among drinkers. The beneficial effect of other dietary components might be countered by a harmful effect of non-heme iron, which was expected to mostly exert its effect, if any, among drinkers.

Supplemental iron showed a positive trend with Type 2 diabetes incidence among women who took more than or equal to $30 \mathrm{mg} / \mathrm{d}$ and the association became stronger among heavier drinkers, in agreement with our hypothesis. We used $30 \mathrm{mg} / \mathrm{d}$ as a cut-off point because, in elderly participants in the Framingham Heart Study cohort [32], the risk of having high iron stores was higher in subjects who took more than or equal to $30 \mathrm{mg} / \mathrm{d}$ supplemental iron than in nonusers. We note that the use of supplemental iron might not increase risk in the presence of iron-deficiency anaemia; however, the baseline iron status of these Iowa women is not known.

It is generally believed that light or moderate alcohol consumption can be helpful for preventing Type 2 
Table 5. Adjusted ${ }^{\mathrm{a}}$ relative risks of Type 2 diabetes according to intake of supplemental iron stratified by alcohol consumption in the Iowa Women's Health Study, 1986 to 1997

Supplemental iron

$0 \mathrm{mg} / \mathrm{d} \quad 1-29 \mathrm{mg} / \mathrm{d} \quad \geq 30 \mathrm{mg} / \mathrm{d}$

A. Joint model across all drinking categories ( $p$ for interaction $=0.47$ )

Non-drinkers

Cases

973

Person-years

Incidence density (per 1000)

Multivariate RR (95\% CI)

$1-14 \mathrm{~g} / \mathrm{d}$ alcohol

Cases

Person-years

Incidence density (per 1000)

Multivariate RR (95\% CI)

$\geq 15$ g/d alcohol

Cases

Person-years

Incidence density (per 1000)

Multivariate RR (95\% CI)

$$
128,044
$$

7.6

Reference

553

121,281

4.6

$0.70(0.63-0.79)$

\section{2}

18,431

3.4

$0.50(0.24-0.87)$
150

24,119

6.2

$0.95(0.74-1.22)$

94

24,655

3.8

$0.71(0.54-0.93)$

10

3529

2.8

$0.46(0.24-0.87)$
45

6318

7.1

$1.14(0.61-2.13)$

28

5739

4.9

$0.97(0.50-1.89)$

6

737

8.1

$1.67(0.64-4.39)$

B. Separate models within drinking categories

Non-drinkers

Multivariate RR (95\% CI)

1.0

$0.91(0.76-1.09)$

$1.01(0.74-1.38)$

$p$ trend $=0.53$

$1-14 \mathrm{~g} / \mathrm{d}$ alcohol

Multivariate RR $(95 \% \mathrm{CI})$

$0.98(0.79-1.23)$

$1.21(0.82-1.78)$

$p$ trend $=0.57$

$\geq 15$ g/d alcohol

Multivariate RR $(95 \% \mathrm{CI})$

1.0

$0.86(0.44-1.69)$

$3.03(1.29-7.12)$

$p$ trend $=0.16$

a Adjusted for age, total energy intake, WHR, BMI, physical activity, cigarette smoking, alcohol consumption, education, marital status, residential area, hormone replacement therapy, animal fat, vegetable fat, cereal fibre, dietary magnesium, dietary non-heme iron and heme iron diabetes [33, 34]. In this study, alcohol (mean consumption: $4.5 \mathrm{~g} / \mathrm{d}$ ) also showed an inverse association with Type 2 diabetes. However, we hypothesized that alcohol consumption could interact with iron because the regular consumption of alcohol can disrupt normal iron metabolism in humans, resulting in the excess deposition of iron in the liver [14]. This could happen irrespective of mechanisms relating the beneficial effect of light or moderate alcohol consumption to the development of Type 2 diabetes. A possible harmful effect of alcohol related with iron metabolism might be only a small part of alcohol's various effects. Thus, a net effect of low or moderate alcohol consumption seems to be beneficial in the risk of diabetes. However, in this study, alcohol consumption was chosen as one of possible situations that can disturb iron metabolism. We speculate that there might be other conditions related with iron metabolism, even though at present they are unknown. Therefore, our results should be interpreted as showing that high iron intake might increase the risk of diabetes under certain situations that can disturb iron metabolism. Our result should not be interpreted to stop drinking alcohol.

The underlying mechanism of the association between alcohol consumption and abnormal iron metabolism is largely unknown; however, it is likely that the two major proteins of iron metabolism, ferritin and transferrin, are intimately involved in the process [14]. The relation between alcohol consumption and disturbance of iron metabolism has mostly been observed among alcoholics [14]; however, recent studies have reported that, even in general populations, there is a positive dose-response relationship between the amount of alcohol consumed and serum ferritin level $[15,16,17]$. For example, those who consumed $10 \mathrm{~g} / \mathrm{d}$ of alcohol had higher ferritin levels than non-drinkers [15]. Therefore, the effect of alcohol on iron metabolism seems to start at a lower level than what was believed. Based on these observations, we hypothesized that, if there were an effect of iron intake on the risk of Type 2 diabetes, it would be a stronger association among drinkers. 
Table 6. Adjusted ${ }^{\mathrm{a}}$ relative risks of Type 2 diabetes according to intake of red meat stratified by alcohol consumption in the Iowa Women's Health Study, 1986 to 1997

Quintile of red meat consumption

\begin{tabular}{lllll}
\hline Q1 & Q2 & Q3 & Q4
\end{tabular}

A. Joint model across all drinking categories $(p$ for interaction $=0.02$ )

Non-drinkers

\begin{tabular}{|c|c|c|c|c|c|}
\hline Cases & 181 & 264 & 229 & 223 & 271 \\
\hline Person-years & 28,293 & 39,433 & 30,510 & 30,693 & 29,537 \\
\hline Incidence density (per 1000) & 6.4 & 6.7 & 7.5 & 7.3 & 9.2 \\
\hline Multivariate RR $(95 \% \mathrm{CI})$ & Reference & $0.97(0.79-1.18)$ & $1.14(0.93-1.40)$ & $1.05(0.85-1.30)$ & $1.10(0.88-1.39)$ \\
\hline \multicolumn{6}{|l|}{$1-14 \mathrm{~g} / \mathrm{d}$ alcohol } \\
\hline Cases & 85 & 145 & 121 & 150 & 174 \\
\hline Person-years & 24,670 & 39,181 & 29,255 & 30,267 & 28,300 \\
\hline Incidence density (per 1000) & 3.4 & 3.7 & 4.1 & 5.0 & 6.1 \\
\hline Multivariate RR $(95 \% \mathrm{CI})$ & $0.65(0.52-0.89)$ & $0.68(0.54-0.85)$ & $0.66(0.52-0.85)$ & $0.84(0.67-1.07)$ & $0.89(0.69-1.13)$ \\
\hline \multicolumn{6}{|l|}{$\geq 15 \mathrm{~g} / \mathrm{d}$ alcohol } \\
\hline Cases & 5 & 23 & 12 & 13 & 25 \\
\hline Person-years & 3806 & 6552 & 4450 & 4225 & 3668 \\
\hline Incidence density (per 1000) & 1.3 & 3.5 & 2.7 & 3.1 & 6.8 \\
\hline Multivariate RR (95\% CI) & $0.27(0.11-0.66)$ & $0.64(0.41-1.00)$ & $0.40(0.22-0.71)$ & $0.43(0.24-0.79)$ & $0.90(0.58-1.41)$ \\
\hline
\end{tabular}

B. Separate models within drinking categories

Non-drinkers

Multivariate RR $(95 \%$ CI) 1

$1-14 \mathrm{~g} / \mathrm{d}$ alcohol

Multivariate RR $(95 \% \mathrm{CI}) \quad 1.0$

$1.01(0.76-1.34) 0.98(0.73-1.33) \quad 1.19(0.89-1.61) 1.19(0.84-1.67)$

$0.98(0.80-1.20) \quad 1.15(0.93-1.42) \quad 1.06(0.85-1.32) \quad 1.11(0.86-1.42) \quad p$ trend $=0.28$

$\geq 15 \mathrm{~g} / \mathrm{d}$ alcohol

Multivariate RR (95\% CI) 1.0

$2.56(0.96-6.84) 1.73(1.57-5.21) \quad 1.89(0.62-5.77) 4.62(1.50-14.21)$

$=0.04$

a Adjusted for age, total energy intake, WHR, BMI, physical activity, cigarette smoking, alcohol consumption, education, marital status, residential area, hormone replacement therapy, animal fat, vegetable fat, cereal fibre, and dietary magnesium

The main food source of heme-iron is red meat [21]; other meat constituents besides iron might explain our findings. Indeed, the association between red meat and Type 2 diabetes was positive and very similar to that for heme iron: as alcohol consumption increased, the positive association became stronger. Some studies have reported a positive association between meat or processed meat intake and Type 2 diabetes $[31,35]$. Saturated fat intake or nitrites were the main interests in these papers, but saturated fat intake was not associated with Type 2 diabetes in most large prospective studies $[31,35,36]$, and the relevance of nitrites or nitrite-derived nitrosamines for Type 2 diabetes is unclear [36].

This study was limited by the lack of biological samples. In addition, measurement of diet and diabetes was imperfect. The reproducibility and validation of the food-frequency questionnaire used in Iowa Women's Health Study was evaluated in 44 study participants [37]. The reproducibility of total dietary iron was similar to that of macronutrients $(r=0.47-0.81)$, whereas the validity compared with five 24-h dietary recalls was very low ( $\mathrm{r}=0.07$ unadjusted for total energy; -0.09 adjusted for total energy). The absolute amount of dietary iron intake measured by the food frequency questionnaire was only half of that of the five 24-h dietary recalls. Most dietary iron is in the form of non-heme iron and an important source of non-heme iron is iron-fortified commercial foods such as breakfast cereal. Thus, a choice of a specific brand or type during the period of dietary recall survey might greatly influence the absolute amount of dietary iron intake, and therefore result in a very low validity because the food frequency questionnaire had limited information on breakfast cereal. Non-heme and heme iron were not reported separately, but the two types of dietary iron might show a different reproducibility and validity because their main food sources, and their validities, are different. Main food sources of heme iron are very limited compared with non-heme iron. There- 
fore, validity of heme iron might be much higher than those of non-heme iron. For example, the validity of heme iron might be similar to that of saturated fat, which has similar food sources with heme iron. Differential validity of measurement between non-heme and heme iron might explain the observed difference in association with incident Type 2 diabetes.

Incident cases of Type 2 diabetes were ascertained by self-report. A validation study of self-reported diabetes was conducted on 85 cohort participants in 1988 after the first follow-up survey. Subjects tend to overreport having diabetes; of 44 women who reported having diabetes at baseline, $28(64 \%)$ were confirmed as having diabetes by their physician [38]. All 41 women who reported not having diabetes at baseline were confirmed as not having diabetes. Non-validated positive reports could nonetheless reflect some level of diabetes. One study [39] found that several people with non-validated positive reports of diabetes had some history of glycosuria. Furthermore, the fasting glucose criterion for diabetes was more than or equal to $140 \mathrm{mg} / \mathrm{dl}$ in 1988 , but has subsequently been lowered to less than or equal to $126 \mathrm{mg} / \mathrm{dl}$. Thus, some women who falsely reported a diagnosis of diabetes might still have some level of underlying disease, such as impaired glucose tolerance. The ascertainment of diabetes in the present study was sensitive enough to confirm associations with other known risk factors for Type 2 diabetes. Assuming that the error in diabetes ascertainment was independent and nondifferential, the present findings would only be strengthened by more accurate ascertainment of disease.

In summary, although obesity is the most important risk factor for Type 2 diabetes, our results suggest that a high dietary heme iron intake and/or supplemental iron is associated with an increased risk of Type 2 diabetes, especially among drinkers. Further studies with biochemical indicators of body iron stores would be desirable to further evaluate this possible interaction among Type 2 diabetes, dietary iron and alcohol intake.

\section{References}

1. Mendler MH, Turlin B, Moirand R et al. (1999) Insulin resistance-associated hepatic iron overload. Gastroenterology 117:1155-1163

2. Tuomainen TP, Nyyssonen K, Salonen R et al. (1997) Body iron stores are associated with serum insulin and blood glucose concentrations. Population study in 1013 eastern Finnish men. Diabetes Care 20:426-428

3. Salonen JT, Tuomainen TP, Nyyssonen K, Lakka HM, Punnonen K (1998) Relation between iron stores and noninsulin dependent diabetes in men: case-control study. BMJ 317:727-730

4. Perez de Nanclares G, Castano L, Gaztambide S, Bilbao JR, Pi J, Gonzalez ML, Vazquez JA (2000) Excess iron storage in patients with type 2 diabetes unrelated to primary hemochromatosis. N Engl J Med 343:890-891
5. Lao TT, Chan PL, Tam KF (2001) Gestational diabetes mellitus in the last trimester-a feature of maternal iron excess? Diabet Med 18:218-223

6. Facchini FS (1998) Effect of phlebotomy on plasma glucose and insulin concentrations. Diabetes Care 21:2190

7. Hua NW, Stoohs RA, Facchini FS (2001) Low iron status and enhanced insulin sensitivity in lacto-ovo vegetarians. Br J Nutr 86:515-519

8. Fernandez-Real JM, Penarroja G, Castro A, GarciaBragado F, Hernandez-Aguado I, Ricart W (2002) Blood letting in high-ferritin type 2 diabetes: effects on insulin sensitivity and beta-cell function. Diabetes 51:1000-1004

9. Phelps G, Chapman I, Hall P, Braund W, Mackinnon M (1989) Prevalence of genetic haemochromatosis among diabetic patients. Lancet 2:233-234

10. Frayling T, Ellard S, Grove J, Walker M, Hattersley AT (1998) C282Y mutation in HFE (haemochromatosis) gene and type 2 diabetes. Lancet 351:1933-1934

11. Fernandez-Real JM, Lopez-Bermejo A, Ricart W (2002) Cross-talk between iron metabolism and diabetes. Diabetes 51:2348-2354

12. Conrad ME, Umbreit JN (2000) Iron absorption and transport-An update. Am J Hematol 64:287-298

13. Ponka P, Beaumont C, Richardson DR (1998) Function and regulation of transferrin and ferritin. Semin Hematol $35: 35-54$

14. Fletcher LM, Halliday JW, Powell LW (1999) Interrelationships of alcohol and iron in liver disease with particular reference to the iron-binding proteins, ferritin and transferrin. J Gastroenterol Hepatol 14:202-214

15. Leggett BA, Brown NN, Bryant SJ, Duplock L, Powell LW, Halliday JW (1990) Factors affecting the concentrations of ferritin in serum in a healthy Australian population. Clin Chem 36:1350-1355

16. Lakka TA, Nyyssönen K, Salonen JT (1994) Higher levels of conditioning leisure time physical activity are associated with reduced levels of stored iron in Finnish men. Am J Epidemiol 140:148-160

17. Milman N, Byg KE, Ovesen L (2000) Iron status in Danes 1994 II: prevalence of iron deficiency and iron overload in 1319 Danish women aged 40-70 years. Influence of blood donation, alcohol intake and iron supplement. Ann Hematol 79:612-621

18. Gapstur SM, Potter JD, Folsom AR (1993) Increased risk of breast cancer with alcohol consumption in postmenopausal women. Am J Epidemiol 136:1221-1231

19. Willett WC, Sampson L, Browne ML et al. (1988) The use of self-administered questionnaire to assess diet four years in the past. Am J Epidemiol 127:188-199

20. Feskanich D, Rimm EB, Giovannucci EL et al. (1993) Reproducibility and validity of food intake measurements from a semiquantitative food frequency questionnaire. J Am Diet Assoc 93:790-796

21. Monsen ER (1988) Iron nutrition and absorption: dietary factors which impact iron bioavailability. J Am Diet Assoc 88:786-790

22. Reif DW (1992) Ferritin as a source of iron for oxidative damage. Free Rad Biol Med 12:417-427

23. Ascherio A, Willett WC, Rimm EB, Giovannucci EL, Stampfer MJ (1994) Dietary iron intake and risk of coronary disease among men. Circulation 89:969-974

24. Kang JO (2001) Chronic iron overload and toxicity: clinical chemistry perspective. Clin Lab Sci 14:209-219

25. Fleming DJ, Jacques PF, Dallal GE, Tucker KL, Wilson PW, Wood RJ (1998) Dietary determinants of iron stores in a free-living elderly population: the Framingham Heart Study. Am J Clin Nutr 67:722-733 
26. Backstrand JR, Allen LH, Black AK, Mata M de, Pelto GH (2002) Diet and iron status of non-pregnant women in rural Central Mexico. Am J Clin Nutr 76:156-164

27. Doyle W, Crawley H, Robert H, Bates CJ (1999) Iron deficiency in older people: interactions between food and nutrient intakes with biochemical measures of iron; further analysis of the National Diet and Nutrition Survey of people aged 65 years and over. Eur J Clin Nutr 53: 552-559

28. Miles CW, Collins JS, Holbrook JT, Patterson KY, Bodwell CE (1984) Iron intake and status of men and women consuming self-selected diets. Am J Clin Nutr 40:1393-1396

29. Van Dam RM, Rim EB, Willett WC, Stampfer MJ, Hu FB (2002) Dietary patterns and risk for type 2 diabetes mellitus in U.S. Men. Ann Intern Med 136:201-209

30. Meyer KA, Kushi LH, Jacobs DR Jr, Folsom AR (2001) Dietary fat and incidence of type 2 diabetes in older Iowa women. Diabetes Care 24:1528-1535

31. Meyer KA, Kushi LH, Jacobs DR Jr, Slavin J, Sellers TA, Folsom AR (2000) Carbohydrates, dietary fiber, and incident type 2 diabetes in older women. Am J Clin Nutr 71:921-930

32. Fleming DJ, Tucker KL, Jacques PF, Dallal GE, Wilson PWF, Wood RJ (2002) Dietary factors associated with the risk of high iron stores in the elderly Framingham Heart Study Cohort. Am J Clin Nutr 76:1375-1384
33. Wannamethee SG, Shaper AG, Perry IJ, Alberti KG (2002) Alcohol consumption and the incidence of type II diabetes. J Epidemiol Community Health 56:542-548

34. Vegt F de, Dekker JM, Groeneveld WJ et al. (2002) Moderate alcohol consumption is associated with lower risk for incident diabetes and mortality: the Hoorn Study. Diabetes Res Clin Pract 57:53-60

35. Dam RM van, Willett WC, Rimm EB, Stampfer MJ, Hu FB (2002) Dietary fat and meat intake in relation to risk of type 2 diabetes in men. Diabetes Care 25:417-424

36. Hu FB, Dam RM van (2001) Diet and risk of type II diabetes: the role of types of fat and carbohydrate. Diabetologia 44:805-817

37. Munger RG, Folsom AR, Kushi LH, Kaye SA, Sellers TA (1992) Dietary assessment of older Iowa women with a food frequency questionnaire: nutrient intake, reproducibility, and comparison with 24-hour dietary recall interviews. Am J Epidemiol 136:192-200

38. Kaye SA, Folsom AR, Sprafka JM, Prineas RJ, Wallace RB (1991) Increased incidence of diabetes mellitus in relation to abdominal adiposity in older women. J Clin Epidemiol 44:329-334

39. Midthjell K, Holmen J, Bjorndal A, Lund-Larsen G (1992) Is questionnaire information valid in the study of a chronic disease such as diabetes?: The Nord-Trondelag Diabetes Study. J Epidemiol Community Health 46:537-542 